Project Title: Engineering Plant One-Carbon Metabolism

Grant\#: Metabolic Engineering Grant (DE-FG02-99ER20344)

P.I.: David Rhodes

Institution: Purdue University

Department: Department of Horticulture and Landscape Architecture

Collaborators, Institutions and their Support:

Andrew Hanson [University of Florida] NSF; Hans Bohnert [University of Arizona] NSF; Douglas A. Gage [Michigan State University] NSF; Yair Shachar-Hill [New Mexico State University] NIST.

Final Progress Report October 1999 - February 2003

\title{
Final Progress Report Summary
}

Primary and secondary metabolism intersect in the one-carbon $\left(\mathrm{C}_{1}\right)$ area. Primary metabolism supplies most of the $\mathrm{C}_{1}$ units and competes with secondary metabolism for their use. This competition is potentially severe because secondary products such as lignin, alkaloids, and glycine betaine (GlyBet) require massive amounts of $\mathrm{C}_{1}$ units. Towards the goal of understanding how $\mathrm{C}_{1}$ metabolism is regulated at the metabolic and gene levels so as to successfully engineer $C_{1}$ supply to match demand, we have: (1) cloned complete suites of $\mathrm{C}_{1}$ genes from maize and tobacco, and incorporated them into DNA arrays; (2) prepared antisense constructs and mutants engineered with alterations in $\mathrm{C}_{1}$ unit supply and demand; and (3) have quantified the impacts of these alterations on gene expression (using DNA arrays), and on metabolic fluxes (by combining isotope labeling, MS, NMR and computer modeling).

Metabolic flux analysis and modeling in tobacco engineered for GlyBet synthesis by expressing choline oxidizing enzymes in either the chloroplast or cytosol, has shown that the choline biosynthesis network is rigid, and tends to resist large changes in $\mathrm{C}_{1}$ demand. A major constraint on engineering enhanced flux to GlyBet in tobacco is a low capacity of choline transport across the chloroplast envelope.

Maize and sorghum mutants defective in GlyBet synthesis show greatly reduced flux of $\mathrm{C}_{1}$ units into choline in comparison to GlyBet-accumulating wildtypes, but this is not associated with altered expression of any of the $\mathrm{C}_{1}$ genes. Control of $\mathrm{C}_{1}$ flux to choline in tobacco, maize and sorghum appears to reside primarily at the level of $N$-methylation of phosphoethanolamine. A candidate signal for the control of this flux is the pool size of phosphocholine which down-regulates and feedback inhibits phosphoethanolamine $N$-methyltransferase.

Methionine $S$-methyltransferase (MMT) catalyzes the synthesis of $S$-methylmethionine (SMM) from methionine (Met) and S-adenosylmethionine (AdoMet). SMM can be reconverted to Met by donating a methyl group to homocysteine, and concurrent operation of this reaction and that mediated by MMT sets up the SMM cycle. The genes encoding the enzymes of the SMM cycle were cloned and characterized during this project. SMM has been hypothesized to be essential as a methyl donor or as a transport form 
of sulfur, and the SMM cycle has been hypothesized to guard against depletion of the free Met pool by excess AdoMet synthesis, or to regulate AdoMet level and hence the AdoMet/S-adenosylhomocysteine ratio (the methylation ratio). To test these hypotheses, we isolated insertional mmt mutants of Arabidopsis and maize. Both mutants lacked the capacity to produce SMM and thus had no SMM cycle. They nevertheless grew and reproduced normally and the seeds of the Arabidopsis mutant had normal sulfur contents. These findings rule out an indispensable role for SMM as a methyl donor or in sulfur transport. The Arabidopsis mutant had significantly higher AdoMet and lower $S$-adenosylhomocysteine (AdoHCy) levels than the wild type, and consequently a higher methylation ratio (20 vs. 14). Free Met and thiol pools were unaltered in this mutant, although there was a 50\% decrease in free threonine (Thr) and changes in other amino acids. These data indicate that the SMM cycle contributes to regulation of AdoMet levels rather than preventing depletion of free Met. Since AdoMet activates Thr synthase, that Thr level was not higher but lower in the mmt mutant implies that AdoMet is sequestered away from Thr synthase, which is chloroplastic.

Results obtained with the Arabidopsis mmt mutant and wildtype have been integrated into a metabolic model of the intersecting methylation, SMM, and methionine salvage cycles. This model adequately accounts for the steady-state pool sizes of Met, SMM, AdoMet and AdoHCy in wildtype, and the small changes in AdoMet and AdoHCy levels associated with knockout of MMT. This model is now being used to predict the time-course of changes in AdoMet, Met, AdoHCy, and SMM mass isotopomers when Arabidopsis is fed with ${ }^{13} \mathrm{C}_{5}$-Met at different doses, and to evaluate the metabolic consequences of knockout of adenosine kinase or AdoHCy hydrolase in Arabidopsis.

\section{Personnel:}

A graduate student (Gregory J. Peel) was recruited to the DOE-funded project at Purdue University. Greg Peel has been a co-author of two of the papers listed below, and is currently preparing manuscripts on the metabolism of ${ }^{14} \mathrm{C}$-formaldehyde in control and salt-stressed maize and sorghum near-isogenic lines with and without GlyBet. Greg Peel is expected to graduate with a Ph.D. in 2004.

\section{Publications resulting from the award:}

1. Nuccio, M.L., Rhodes, D., McNeil, S.D., and Hanson, A.D. (1999) Metabolic engineering of plants for osmotic stress resistance. Curr. Opin. Plant Biol. 2: 128-134.

2. McNeil, S.D., Nuccio, M.L., Rhodes, D., Shachar-Hill, Y., and Hanson, A.D. (2000). Radiotracer and computer modeling evidence that phosphobase methylation is the main route of choline synthesis in tobacco. Plant Physiol. 123: 371-380.

3. Ranocha, P., Bourgis, F., Ziemak, M.J., Rhodes, D., Gage, D.A., Hanson, A.D. (2000)

Characterization and functional expression of cDNAs encoding methionine-sensitive and -insensitive homocysteine S-methyltransferases from Arabidopsis. J. Biol. Chem. 275: 15962-15968.

4. McNeil, S.D., Rhodes, D., Russell, B.L., Nuccio, M.L., Shachar-Hill, Y., Hanson, A.D. (2000). Metabolic modeling identifies key constraints on an engineered glycine betaine synthesis pathway in tobacco. Plant Physiol. 124: 153-162.

5. Morgan, J.A., Rhodes, D. (2002). Mathematical modeling of plant metabolic pathways. Metabolic 
Engineering 4: 80-89.

6. Wood, K.V., Bonham, C.C., Miles, D., Rothwell, A.P., Peel, G., Wood, B.C., Rhodes, D. (2002) Characterization of betaines using electrospray MS/MS. Phytochemistry 59: 759-765.

7. Rhodes, D., McNeil, S.D., Nuccio, M.L., Hanson, A.D. (2004) Metabolic engineering and flux analysis of glycine betaine synthesis in plants: progress and prospects. In (B.N. Kholodenko, H.V. Westerhoff, eds.) "Metabolic Engineering in the Post Genomic Era", Horizon Scientific Press, Wymondham, Norfolk, U.K. pp. 409-434.

8. Kocsis, M.G., Ranocha, P., Gage, D.A., Simon, E.S., Rhodes, D., Peel, G.J., Mellema, S., Saito, K., Awazuhara, M., Li, C., Meeley, R.B., Tarczynski, M.C., Wagner, C., Hanson, A.D. (2003) Insertional inactivation of the methionine $S$-methyltransferase gene eliminates the $S$-methylmethionine cycle and increases the methylation ratio. Plant Physiol. 131: 1808-1815.

9. Yang, W.-J., Rich, P.J., Axtell, J.D., Wood, K.V., Bonham, C.C., Ejeta, G., Mickelbart, M.V., Rhodes, D. (2003) Genotypic variation for glycinebetaine in sorghum. Crop Science 43: 162-169.

10. Mickelbart, M.V., Peel, G., Joly, R.J., Rhodes, D., Ejeta, G., Goldsbrough, P.B. (2003) Development and characterization of near-isogenic lines of sorghum segregating for glycinebetaine accumulation. Physiologia Plantarum 118: 253-261.

11. Rhodes, D., Peel, G.J., Dudareva N. (2004) Engineering pathways of secondary metabolism. In (R.M. Goodman, ed.) "Encyclopedia of Plant and Crop Science", Marcel Dekker, N.Y. pp. 720-723

Featured scientific articles published: None

Patents obtained based on DOE-supported work: None

Honors or specific recognition: None

Collaborations resulting from this award, including interactions (formal and informal) you currently have with the industrial sector:

Rhodes has initiated a collaboration with Dr. Barbara Moffatt, Department of Biology, University of Waterloo, Ontario, Canada, to apply mathematical modeling of stable isotope fluxes via the transmethylation cycle to transgenic Arabidopsis expressing antisense adenosine kinase and $S$ adenosylhomocysteine hydrolase.

Rhodes has initiated a collaboration with Dr. John A. Morgan, a new faculty member in the Dept. of Chemical Engineering at Purdue University, in the area of metabolic pathway modeling and flux analysis in plants.

Rhodes has initiated a collaboration with Dr. Natalia Dudareva (Department of Horticulture and Landscape Architecture, Purdue University) on 1-C metabolism involvement in floral scent production. 
Emphasis is on the role of the transmethylation cycle in providing methyl groups for synthesis of methyl benzoate in snapdragon and petunia flowers.

Rhodes has provided seed of near-isogenic glycine betaine-containing (Bet1/Bet1) and glycine betainedeicient (bet1/bet1) maize lines to Dr. Kevin Simcox, Pioneer Hi-bred International Inc, Johnston, Iowa, to facilitate progress in a study on the role of glycine betaine in Fusarium infection in maize.

\section{Outreach:}

As part of the educational mission of our "Engineering Plant One-Carbon Metabolism” grant we proposed to develop a www site that would provide a tutorial on simulation of metabolism, that provides a range of examples of metabolic flux analysis, references to modeling literature, and which offers free metabolic modeling software. The URL for this site is:

http://www.hort.purdue.edu/cfpesp/models/models.htm

We further proposed that we would hold computer modeling workshops to encourage the further use of these tools. Accordingly we offered a one-day workshop on computer modeling of metabolism that was held at New Mexico State University on January 7, 2000 as part of a symposium on "Quantifying and engineering of plant metabolism” [Jan 7 - Jan 8, 2000]. The workshop was attended by 25 graduate students in the molecular biology program at NMSU during a morning session, and by 20 senior scientists in an afternoon session. Each workshop participant was guided through the use of models for simulating radioisotope tracer kinetics, including in the use of dynamic kinetic models. A similar workshop was held at the ASPP meeting in San Diego in July, 2000. The latter workshop was comprised of a series of three 30-minute talks on the evening of the first day of the meeting (July 16), followed by 3 two-hour "hands-on" computer modeling training sessions on July $17-19$ (12:00 noon - 2:00 PM). A total of 36 plant scientists participated in these "hands-on" computer modeling training sessions. 